\author{
$\mathrm{B}, \mathrm{N}$ 共掺杂的 $\ln _{2} \mathrm{O}_{3} / \mathrm{TiO}_{2}$ 制备与光催化产氢性能研究 \\ 李宸 ${ }^{a, b}$ 陈凤华 $*, a$ 叶丽 ${ }^{a}$ 李伟 $a, b$ 于晗 ${ }^{a}$ 赵泮 $*, a, b$ \\ ( ${ }^{a}$ 中国科学院化学研究所 极端环境高分子材料重点实验室 北京 100190) \\ ${ }^{b}$ 中国科学院大学 化学科学学院 北京 100190)
}

\begin{abstract}
摘要 为拓宽 $\mathrm{TiO}_{2}$ 的光吸收范围以及提高光生载流子的利用率, 本工作利用 $\mathrm{B}, \mathrm{N}$ 共掺杂改性的方法, 通过聚合物前驱 体法设计并制备了 $\mathrm{In}_{2} \mathrm{O}_{3}$ 改性的 $\mathrm{TiO}_{2}$ 光催化剂. 在前驱体合成中引入了聚乙二醇( $\mathrm{PEG}$ )作为致孔剂. PEG 在前驱体转化 为无机氧化物的热处理过程中分解离去、形成介孔等不同尺度的孔隙, 提高了样品的比表面积. 异质结结构在产物中 得到有效构筑，带隙宽度由 P25 的 $3.09 \mathrm{eV}$ 缩窄至 $2.71 \mathrm{eV}$ (样品 IT-500, $500{ }^{\circ} \mathrm{C}$ 退火产物). $\mathrm{B}, \mathrm{N}$ 掺杂进入 $\mathrm{TiO}_{2}$ 晶格内, 形 成了 Ti-N-B 和 Ti-O-B 结构, 同时也存在 $\mathrm{N}$ 的填隙掺杂, 有助于带隙的缩窄、并拓宽可见光吸收范围. $\mathrm{In}_{2} \mathrm{O}_{3} / \mathrm{TiO}_{2}$ 异质 结结构的构筑, 促进了电子-空穴对的分离与转移, 提高了光生载流子的利用率. 在大于 $380 \mathrm{~nm}$ 可见光的照射下, 样品 IT-500 的可见光催化产氢速率达到了 $5961 \mu \mathrm{mol} \cdot \mathrm{g}^{-1} \cdot \mathrm{h}^{-1}$, 催化剂经过分离回收后进行循环实验, 仍能保持良好的光催 化活性. 为了进一步提高其回收性, 利用气纺丝制备了 $\mathrm{B}, \mathrm{N}$ 掺杂的 $\mathrm{In}_{2} \mathrm{O}_{3} / \mathrm{TiO}_{2}$ 纳米纤维棉, 在最佳焙烧温度 $500{ }^{\circ} \mathrm{C}$ 下, 所获得的纤维棉状光催化剂的氢气产生速率达到 $1186 \mu \mathrm{mol} \cdot \mathrm{g}^{-1} \cdot \mathrm{h}^{-1}$, 纤维棉简化了回收再利用的过程, 经过 5 次循环 实验后仍能达到初始产氢速率的 $97 \%$.
\end{abstract}

关键词 光催化; $\mathrm{TiO}_{2}$; 非金属元素共掺杂; 异质结; 可见光响应; 纳米纤维

\title{
Preparation and Photocatalytic Hydrogen Production of B, N Co-doped $\mathrm{In}_{2} \mathrm{O}_{3} / \mathrm{TiO}_{2}$
$\mathrm{Li}, \mathrm{Chen}{ }^{a, b}$
Chen, Fenghua ${ }^{*, a}$
$\mathrm{Ye}, \mathrm{Li}^{a}$
$\mathrm{Li}, \mathrm{Wei}^{a, b}$
$\mathrm{Yu}, \operatorname{Han}^{a}$
Zhao, Tong ${ }^{*, a, b}$ \\ ( ${ }^{a}$ Key Laboratory of Science and Technology on High-tech Polymer Materials, Institute of Chemistry Chinese Academy of Sciences, Beijing 100190, China) \\ ( ${ }^{b}$ School of Chemical Sciences, University of Chinese Academy of Sciences, Beijing 100190, China)
}

\begin{abstract}
In order to improve light absorption range of $\mathrm{TiO}_{2}$ and utilization rate of photogenerated carriers, we use $\mathrm{B}, \mathrm{N}$ co-doping and $\mathrm{In}_{2} \mathrm{O}_{3}$ blending to modify the $\mathrm{TiO}_{2}$ photocatalyst. Sample preparation is conducted through polymer precursor method and uniform distribution of the components is ensured. Polyethylene glycol (PEG) is added at the beginning of sample preparation and removed during the annealing process at high temperatures. X-ray diffraction (XRD), scanning electron microscope (SEM), high-resolution transmission microscope (HRTEM), specific surface area and pore structure analyzer, X-ray photoelectron spectroscopy (XPS), ultraviolet-visible absorption spectrum and photoluminescence (PL) spectroscopy are used to characterize the products obtained. $\mathrm{B}$ and $\mathrm{N}$ elements have been detected in the lattice of $\mathrm{TiO}_{2}$. Heterojunction structure of $\mathrm{In}_{2} \mathrm{O}_{3}$ and $\mathrm{TiO}_{2}$ are also observed. Formation of Ti-N-B and Ti-O-B structure is exhibited in this system. Interstitial doping of $\mathrm{N}$ is also observed. These factors contribute to narrow the band gap from $3.09 \mathrm{eV}$ of P25 to $2.71 \mathrm{eV}$ of IT-500 (the modified sample annealed at $500{ }^{\circ} \mathrm{C}$ ). With the introduction and pyrolysis of porogen PEG, mesoporous structure is successfully constructed. Visible light absorption range has been greatly broadened in this modified $\mathrm{TiO}_{2}$ based material. Utilization rate of photogenerated carriers has also been enhanced. When the catalyst is used in the photocatalytic hydrogen production experiment, under the irradiation of visible light $(>380 \mathrm{~nm})$, hydrogen production rate of IT-500 reaches $5961 \mu \mathrm{mol} \bullet$ $\mathrm{g}^{-1} \cdot \mathrm{h}^{-1}$, which is far superior to commercial $\mathrm{TiO}_{2}$ and most of the $\mathrm{TiO}_{2}$ prepared by single modification method. The hydrogen production rate is maintained in the 5-circle test after the catalyst is separated and recycled. When the $\mathrm{B}, \mathrm{N}-\mathrm{In}_{2} \mathrm{O}_{3} / \mathrm{TiO}_{2}$ polymer precursor is gas sprayed, which uses polyvinylpyrrolidone as spinning aid, ethanol and acetic acid as solvents, nanofiber sponge can be obtained and used for hydrogen production. Hydrogen production rate of this material reaches 1186 $\mu \mathrm{mol} \cdot \mathrm{g}^{-1} \cdot \mathrm{h}^{-1}$ and keeps $97 \%$ after 5 -cycle test, which shows high potential for commercial use of this material.

Keywords photocatalysis; $\mathrm{TiO}_{2}$; Co-doping of non-metallic elements; heterojunction; visible-light response; nanofiber
\end{abstract}

* E-mail: fhchen@iccas.ac.cn; tzhao@iccas.ac.cn

Received July 20, 2020; published November 3, 2020.

Supporting information for this article is available free of charge via the Internet at http://sioc-journal.cn.

Project supported by the National Natural Science Foundation of China (No. 21604090).

项目受国家自然科学基金(No. 21604090)资助. 


\section{1 引言}

自从 1972 年 Fujishima 与 $\mathrm{Honda}^{[1]}$ 发表了 $\mathrm{TiO}_{2}$ 作为 光催化剂实现光电催化分解水产氢/氧的工作以来, 光 催化制氢一直被认为是制备氢气这种清洁能源最有潜 力的方法之一 ${ }^{[2]}$. 由于 $\mathrm{TiO}_{2}$ 具有出色的生物、化学稳定 性与光化学稳定性以及合适的电势电位, 因此受到了科 研人员的广泛关注, 其在光催化降解有机污染物 ${ }^{[3-5]}$ 、光 催化制氢 ${ }^{[6-9]}$ 、有害气体的探测与净化领域 ${ }^{[10-11]}$ 都有潜在 的应用前景. 然而由于 $\mathrm{TiO}_{2}$ 本征带隙较宽 $\left(E_{\mathrm{g}}=3.1 \sim 3.2\right.$ $\mathrm{eV}$ ), 只对紫外光有响应, 而对可见光部分几乎毫无响 应，这限制了其在实际应用中对太阳能的高效利用 ${ }^{[12]}$; 同时, $\mathrm{TiO}_{2}$ 吸收光后激发产生的载流子一电子和空穴容 易发生复合, 使得可以传输到催化剂表面参与反应的载 流子数目减少, 降低催化效率 ${ }^{[13]}$. 因此, 缩短 $\mathrm{TiO}_{2}$ 基光 催化剂的带隙, 使其对光谱的吸收拓展至可见光范围 内, 以及解决大量电子-空穴对发生复合的问题, 是 $\mathrm{TiO}_{2}$ 基光催化剂走向应用的关键. 研究人员发现, 通过 调控 $\mathrm{TiO}_{2}$ 的微观形貌与结构, 可以提高半导体的光吸 收率, 例如暴露特定的晶面 ${ }^{[14]}$, 制备多孔结构使得照 射光在材料表面产生多次反射-吸收现象 ${ }^{[15]}$. 此外, 通 过元素掺杂的方式可以有效调节 $\mathrm{TiO}_{2}$ 的能带结构, 如 掺杂 $\mathrm{Fe}, \mathrm{Co}, \mathrm{Cu}, \mathrm{Ni}$ 等金属元素 ${ }^{[16-18]}$, 掺杂 $\mathrm{N}^{[19]}, \mathrm{B}^{[20-22]}$, $\mathrm{C}^{[23-24]}, \mathrm{F}^{[25-27]}, \mathrm{S}^{[28]}$ 等非金属元素. 其中, 非金属元素的 掺杂主要是通过将掺杂元素的 $p$ 轨道与 $\mathrm{TiO}_{2}$ 中 $\mathrm{O} 2 \mathrm{p}$ 轨 道混合, 将价带上移以缩短带隙宽度, 同时降低生成复 合中心的概率, 因此非金属元素的掺杂可以有效地拓宽 $\mathrm{TiO}_{2}$ 对于太阳光特别是可见光波段的吸收范围. 构建异 质结结构可以显著提高电子空穴对的分离效率, 使两种 光生载流子分别在不同的反应位点发生氧化还原反 应 ${ }^{[29]}$, 多种非金属元素共掺杂生成的键合结构也有助 于载流子的分离 ${ }^{[21]}$.

大多数 $\mathrm{TiO}_{2}$ 光催化剂是基于粉末、颗粒状悬浊液 来参与光催化反应 ${ }^{[00-34]}$, 一方面粉末颗粒有聚集趋势, 另一方面在后续处理、分离回收时有诸多不便, 不利于 降低成本. 将 $\mathrm{TiO}_{2}$ 粉末负载在其他载体上, 如玻璃、活 性炭、钢丝网或高分子材料上 ${ }^{[35-36]}$, 由于借助载体成型, 催化剂在使用后仍能维持形态, 便于分离回收与重复利 用. 但负载型催化剂也存在一定缺陷 ${ }^{[37]}$ : 由于载体的 “阴影效应”, 光的穿透被抑制, 这影响了光的利用效率; 同时, 比表面积的减小以及污染物和牺牲剂在整个基材 上的传质减少会降低 $\mathrm{TiO}_{2}$ 在载体上的光催化效率; 另 外, 在液体的流动环境中, 负载型催化剂面临着催化剂 脱落的问题, 使得其在长期使用过程中催化效率逐渐降 低.

为了解决上述问题, 聚合物前驱体法是一种有效的 手段, 聚合物前驱体法是制备陶瓷材料和改性催化剂的 一种新型方法 ${ }^{[3,38]}$, 相比传统方法, 该方法具有如下显 著优点: (1)结构和组成可调, 可以与多种改性方式兼容;
(2)合成条件温和，合成周期短; (3)容易与软模板结合, 煅烧后可形成介孔结构, 增大比表面积; (4)液相前驱体 易于加工成型，方便制备涂层或者纤维. 因此，采用聚 合物前驱体法制备纳米纤维有望解决粉体催化剂分离 回收困难以及负载型催化剂的脱落等问题.

在本工作中, 我们利用聚合物前驱体法, 结合非金 属元素掺杂、构建异质结、引入致孔剂等多种改性手段, 来协同改性 $\mathrm{TiO}_{2}$, 成功制备出一种高效稳定的 $\mathrm{B}, \mathrm{N}$ 共 掺杂的 $\mathrm{In}_{2} \mathrm{O}_{3} / \mathrm{TiO}_{2}$ 光催化剂, 并且利用气纺丝装置制备 了该体系的纤维棉光催化剂. 在可见光照射下, 改性催 化剂粉体和纤维棉状催化剂均表现出优异的光催化产 氢活性, 且纤维棉状催化剂可以极大程度简化分离回收 的过程, 表现出了较大的应用潜力.

\section{2 结果与讨论}

\section{$2.1 \mathrm{~B}, \mathrm{~N}-\mathrm{In}_{2} \mathrm{O}_{3} / \mathrm{TiO}_{2}$ 晶体结构及微观形貌表征}

样品通过聚合物前驱体法制备后煅烧获得. 以钛酸 异丙酯为钛源, 硝酸铟为铟源, 硼酸为硼源, 乙酰胺为 氮源, PEG 为致孔剂, 经可控水解聚合生成 $\mathrm{B}, \mathrm{N}$ 共掺杂 $\mathrm{In}_{2} \mathrm{O}_{3} / \mathrm{TiO}_{2}$ 聚合物前驱体, 将前驱体于不同温度(450、 $500 、 550{ }^{\circ} \mathrm{C}$ )下裂解处理 $30 \mathrm{~min}$, 得到 $\mathrm{B}, \mathrm{N}$ 共掺杂 $\mathrm{In}_{2} \mathrm{O}_{3} / \mathrm{TiO}_{2}$ 催化剂, 根据裂解温度不同, 所得催化剂分 别命名为 IT-450、IT-500、IT-550. 利用 X-射线衍射 (XRD)表征了样品的晶体结构, 如图 1 所示. 由图可得, 不同温度所得的 $\mathrm{IT}^{-} \mathrm{TiO}_{2}$ 样品的晶相均为锐铁矿相, 在 $25.4^{\circ} 、 37.9^{\circ} 、 48.1^{\circ} 、 54.0^{\circ} 、 55.1^{\circ}$ 和 $62.8^{\circ}$ 处的衍射峰, 分 别对应于锐钣矿相 $\mathrm{TiO}_{2}$ 的(101), (004), (200), (105), (211)和(204)晶面(PDF No.21-1272, JCPDS), 此外, 掺杂 样品的 XRD 图在 $2 \theta=30.3^{\circ}$ 处均有一个相对较小的峰, 根据体系的元素组成判断该峰应为 $\mathrm{B}_{2} \mathrm{O}_{3}$ 和 $\mathrm{In}_{2} \mathrm{O}_{3}$ 布拉格 衍射峰的重叠峰, 另外 $\mathrm{B}_{2} \mathrm{O}_{3}$ 的峰比标准谱 (PDF No.06-0297, JCPDS)向小角度偏移, $\mathrm{B}_{2} \mathrm{O}_{3}$ 衍射峰的半峰 宽较大, 强度较弱, 因此结晶性较弱, 晶体结构并不明 显.

通过扫描电子显微镜(SEM) 与高分辨透射电子显微 镜(HRTEM)表征了样品 IT-500 的微观形貌, 由于各样品

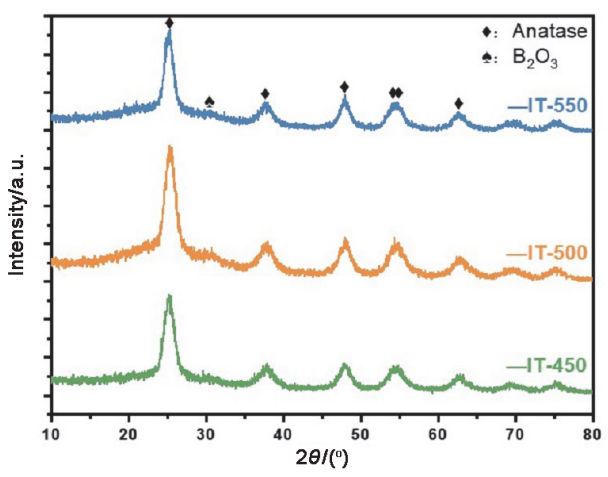

图 1 IT-450, IT-500 和 IT-550 的 XRD 图

Figure 1 XRD patterns of IT-450, IT-500 and IT-550 
的形貌差别不大, 这里仅给出 IT-500 的形貌图. 由 $\operatorname{SEM}$ (图 2A) 可以看出, 所制备的 $\mathrm{B}, \mathrm{N}$ 共掺杂的 $\mathrm{In}_{2} \mathrm{O}_{3} / \mathrm{TiO}_{2}$ 催化剂由粒径均一的纳米颗粒组成, 呈葡萄 状堆积, 粒径在 $10 \sim 20 \mathrm{~nm}$ 左右, 与商品化 $\mathrm{TiO}_{2} \mathrm{P} 25$ 微 观尺寸基本一致 ${ }^{[39]}$. 图 2B $2 \mathrm{D}$ 为样品的高分辨透射电 镜图, 由 HRTEM(图 2D)可以清楚地观察到两种尺寸的 晶格条纹, 其中 $0.41 \mathrm{~nm}$ 的晶格条纹, 为立方 $\mathrm{In}_{2} \mathrm{O}_{3}(211)$ 晶面 ${ }^{[40]}$, 而 $0.35 \mathrm{~nm}$ 的晶格条纹间距归属于锐钛矿相 $\mathrm{TiO}_{2}$ 的(101)晶面 ${ }^{[41]}$, 两种晶格条纹区域紧密接触(图 2D 与图 S1, 见 Supporting Information), 证明成功构建了预 期的 $\mathrm{In}_{2} \mathrm{O}_{3} / \mathrm{TiO}_{2}$ 异质结结构 ${ }^{[4]}$. 各元素在催化剂上分布 均匀, 粉体催化剂的光电子能谱分析(EDS)结果见图 S2.
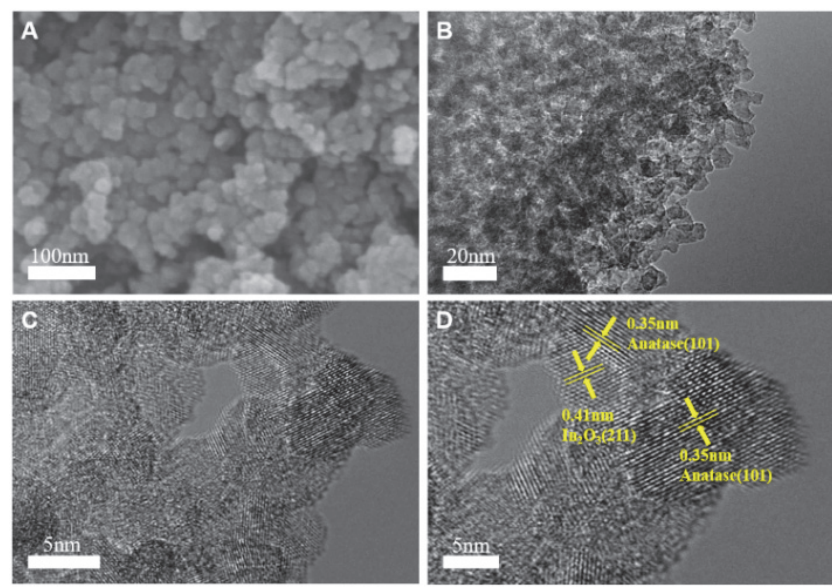

图 2 (A) IT-500 的 SEM 图和(B $\sim \mathrm{D})$ IT-500 的 HRTEM 图

Figure 2 (A) SEM image of IT-500 and $(B \sim D)$ HRTEM images of IT-500

利用 BET 来表征样品的比表面积以及孔径信息. 表 1 列出了三种样品的比表面积以及孔的结构特性, IT-450, IT-500, IT-550 的比表面积分别为 169.9118, $173.4842,140.0745 \mathrm{~m}^{2} / \mathrm{g}$, 而相同条件下测定的 P25 的比 表面积为 $60.8553 \mathrm{~m}^{2} / \mathrm{g}$. 根据 $\mathrm{N}_{2}$ 吸附脱附曲线(图 3A) 可知, 三种样品均呈现 IV 型等温线, 说明均为介孔材 料. 图 3B 显示三种样品的孔径主要分布在 4 7 $\mathrm{nm}$ 之 间. 可见添加致孔剂 PEG, 在煅烧裂解过程中能形成介 孔结构, 根据文献报道, 介孔允许光在其孔道内散射, 大幅提高了样品的比表面积, 从而提高了光吸收能 力 $^{[25]}$, 比表面积的增大有利于提高光催化活性 ${ }^{[42]}$.

\section{$2.2 \mathrm{~B}, \mathrm{~N}-\mathrm{In}_{2} \mathrm{O}_{3} / \mathrm{TiO}_{2}$ 的化学结构表征}

利用光电子能谱 XPS 来表征 IT-500 的组成元素以 及元素所处的化学环境, 样品的 XPS 全谱图见图 S3. 如

表 1 不同样品的 BET 比表面积

Table 1 BET surface areas of different samples

\begin{tabular}{cccc}
\hline 样品名称 & $\begin{array}{c}\text { BET 比表面积/ } \\
\left(\mathrm{m}^{2} \cdot \mathrm{g}^{-1}\right)\end{array}$ & 孔径尺寸 $/ \mathrm{nm}$ & 孔体积 $/\left(\mathrm{cm}^{3} \cdot \mathrm{g}^{-1}\right)$ \\
\hline IT-450 & 169.9118 & 4.7412 & 0.2133 \\
IT-500 & 173.4842 & 5.5640 & 0.2413 \\
IT-550 & 140.0745 & 6.6110 & 0.2315 \\
\hline
\end{tabular}
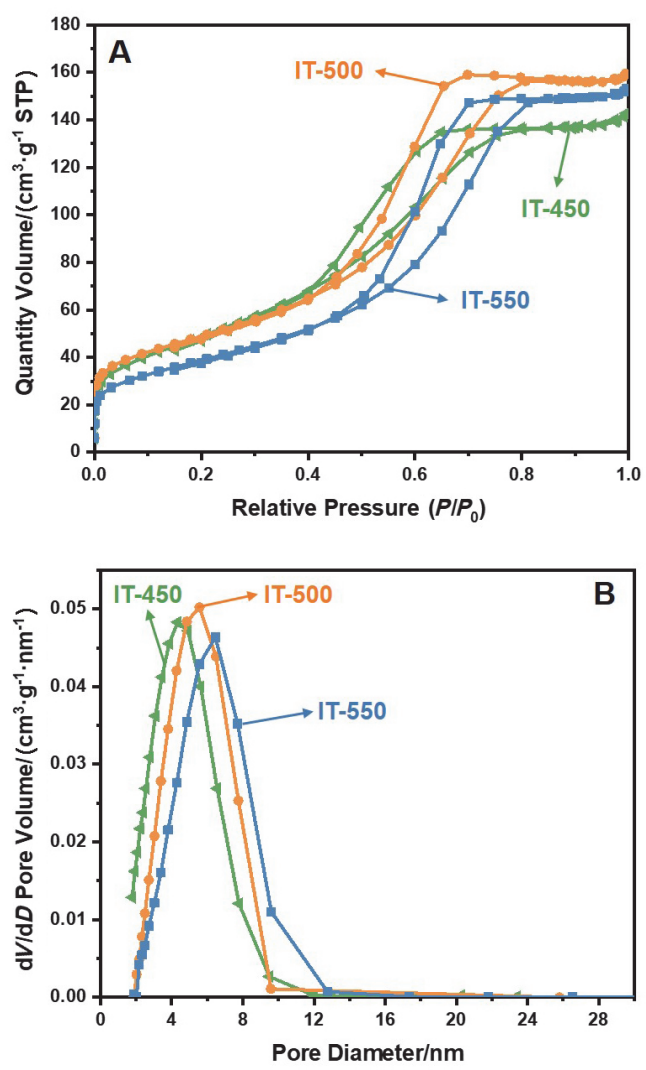

图 3 (A) IT-450, IT-500, IT-550 的 $\mathrm{N}_{2}$ 吸附脱附曲线(77K); (B) IT-450, IT-500 与 IT-550 的 BJH 孔径分布图

Figure 3 (A) $\mathrm{N}_{2}$ absorption-desorption isotherms of IT-450, IT-500 and IT-550; (B) BJH pore size distribution plots of IT-450, IT-500 and IT-550

图 4A 所示, In 3d 的高分辨 XPS 谱图(图 4A)去卷积后, 可以得到属于 In $3 \mathrm{~d}_{5 / 2}(444.80 \mathrm{eV})$ 和 In $3 \mathrm{~d}_{3 / 2}(452.55 \mathrm{eV})$ 的两个特征峰, 特征峰的位置与 $\mathrm{In}_{2} \mathrm{O}_{3}$ 一致, 未出现偏 移, 表明体系中不存在 In-B, In-N 或者 In-Ti 结构. 谱图 中未出现金属铟的 $\operatorname{In} 3 \mathrm{~d}_{5 / 2}$ 信号(结合能 $443.6 \mathrm{eV}$ ), 表明 体系中不存在金属铟, 铟仅以 $\mathrm{In}_{2} \mathrm{O}_{3}$ 形态存在于体系 中 $^{[43]}$. Ti $2 \mathrm{p}$ 高分辨 XPS 谱图(图 4B)中, Ti $2 \mathrm{p}_{1 / 2}$ 的结合能 位于 $464.51 \mathrm{eV}, \mathrm{Ti} 2 \mathrm{p}_{3 / 2}$ 的结合能位于 $458.81 \mathrm{eV}$, 两个特 征峰的结合能差值为 $5.70 \mathrm{eV}$, 表明 $\mathrm{In}_{2} \mathrm{O}_{3} / \mathrm{TiO}_{2}$ 异质结体 系中 $\mathrm{Ti}$ 为 $\mathrm{Ti}^{4+}$. 文献所报道的 $\mathrm{In}_{2} \mathrm{O}_{3} / \mathrm{TiO}_{2}$ 异质结体系中 Ti $2 p_{1 / 2}$ 和 Ti $2 p_{3 / 2}$ 的特征峰分别位于 $464.2 \mathrm{eV}$ 和 458.5 $\mathrm{eV}^{[44]}$, 相比之下, 本工作中样品 $\mathrm{Ti} 2 \mathrm{p}_{1 / 2}$ 和 $\mathrm{Ti} 2 \mathrm{p}_{3 / 2}$ 均降 低了 $0.31 \mathrm{eV}$, 结合能的移动表明 $\mathrm{Ti}$ 与掺杂元素发生相 互作用, 掺杂元素进入到 $\mathrm{TiO}_{2}$ 的晶格内, 提高了 $\mathrm{Ti}$ 的电 子密度 ${ }^{[45]}$. 如图 $4 \mathrm{C}$ 所示, $\mathrm{N} 1 \mathrm{~s}$ 的高分辨 XPS 光谱中, 在 $399.52 \mathrm{eV}$ 处有一个突出的特征峰, 对应于 $\mathrm{TiO}_{2}$ 晶格中 的 Ti-N-B ${ }^{[46]}$ 和以填隙方式掺杂的 $\mathrm{N}$ 结构 ${ }^{[47]}$. 图 4D 显示 了 B 1s 的特征峰位于 $192.01 \mathrm{eV}$ 和 $193.79 \mathrm{eV}$, 分别对应 于 Ti-O-B 结构 ${ }^{[48]}$ 和 $\mathrm{B}_{2} \mathrm{O}_{3}{ }^{[49]}, \mathrm{B}_{2} \mathrm{O}_{3}$ 峰信号的存在与 XRD 的结果一致. 高分辨 XPS 谱图表明, $\mathrm{B}$ 和 $\mathrm{N}$ 的掺杂在一 定程度上影响了 $\mathrm{In}_{2} \mathrm{O}_{3} / \mathrm{TiO}_{2}$ 中 $\mathrm{Ti}$ 的化学环境, 体系中有 Ti-N-B 和 Ti-O-B 结构的形成, 此外也存在以填隙方式 
掺杂的 $\mathrm{N}$ 结构, 该结构有助于提高 $\mathrm{TiO}_{2}$ 的光吸收能 力 $^{[50]}$.

\section{$2.3 \mathrm{~B}, \mathrm{~N}-\mathrm{In}_{2} \mathrm{O}_{3} / \mathrm{TiO}_{2}$ 的光学性质表征}

通过紫外可见吸收光谱图可以对比不同样品对于 光子的吸收范围, 如图 $5 \mathrm{~A}$ 所示, 不同耘烧温度所得的 $\mathrm{B}, \mathrm{N}$ 共掺杂 $\mathrm{In}_{2} \mathrm{O}_{3} / \mathrm{TiO}_{2}$ 催化剂在波长大于 $380 \mathrm{~nm}$ 的可 见光区域吸收范围获得了不同程度的拓展, 样品 IT-500 可见光范围吸收红移最多.

利用 Tauc 方程(1)计算了不同样品的带隙宽度,

$$
(\alpha h v)^{1 / 2}=k^{*}\left(h v-E_{\mathrm{g}}\right)
$$

其中 $\alpha$ 为光吸收系数, $h$ 为普朗克常数, $v$ 为光波频率, $k$ 为与材料性质有关的常数, $E_{\mathrm{g}}$ 为样品的带隙宽度.

如图 5B 所示, 商品化 P25 的带隙为 $3.09 \mathrm{eV}, \mathrm{B}, \mathrm{N}$ 共掺杂及引入 $\mathrm{In}_{2} \mathrm{O}_{3} / \mathrm{TiO}_{2}$ 异质结后, $\mathrm{TiO}_{2}$ 的带隙宽度明 显缩短, 尤其是 $500{ }^{\circ} \mathrm{C}$ 煅烧所得的 IT-500 样品的带隙 宽度仅为 $2.71 \mathrm{eV}$, 表明 $500{ }^{\circ} \mathrm{C}$ 为制备该体系催化剂的 最佳温度. 结合 XPS 表征数据, 可知改性样品中的 Ti-N-B 结构、Ti-O-B 结构与填隙方式掺杂的 N 对带隙 变窄做出了贡献.
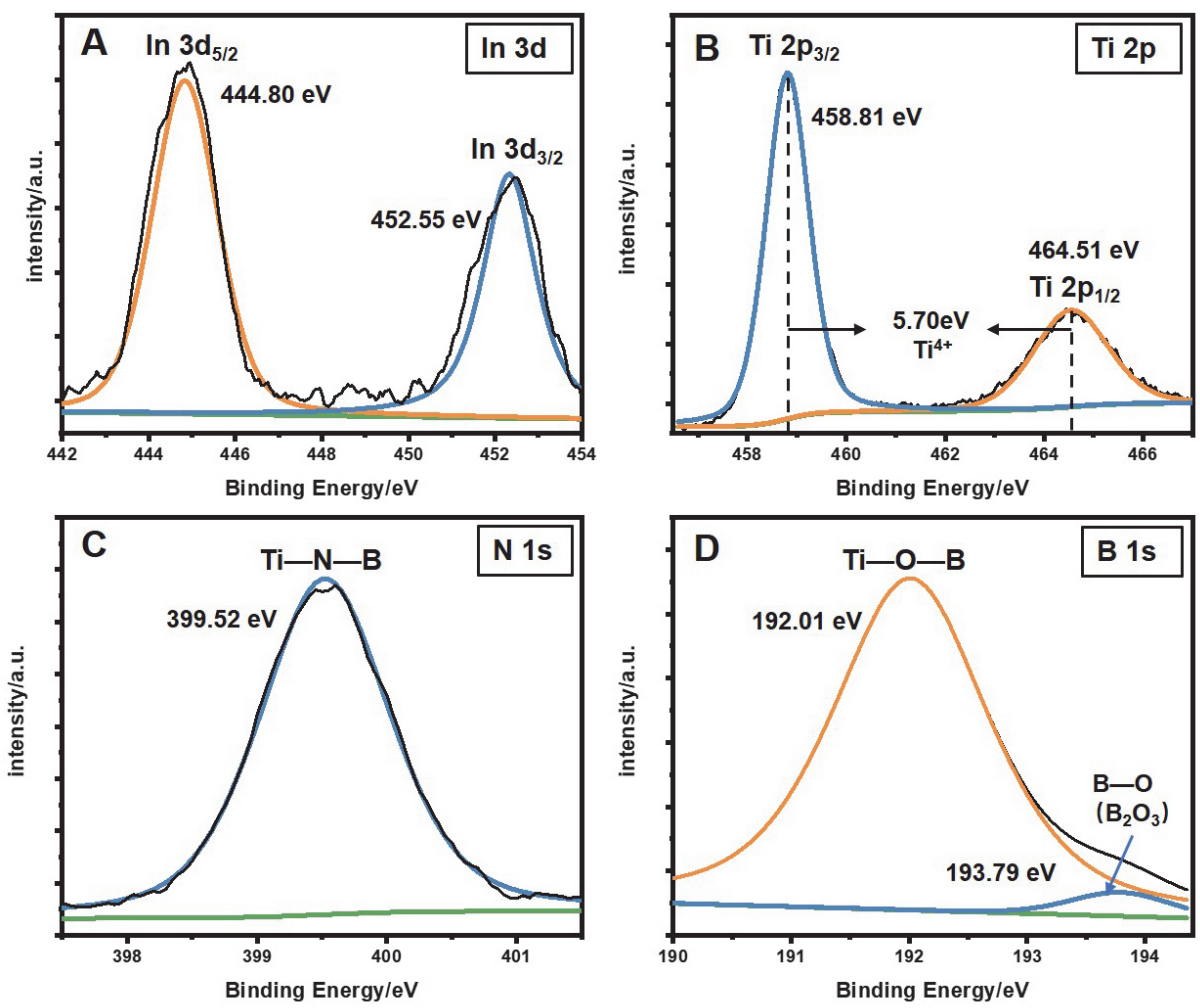

图 4 样品 IT-500 的高分辨 In 3d (A), Ti 2p (B), N 1s (C)和 B 1s (D) XPS 谱图

Figure 4 Fitted XPS spectra of In 3d (A), Ti 2p (B), N 1s (C) and B 1s (D)
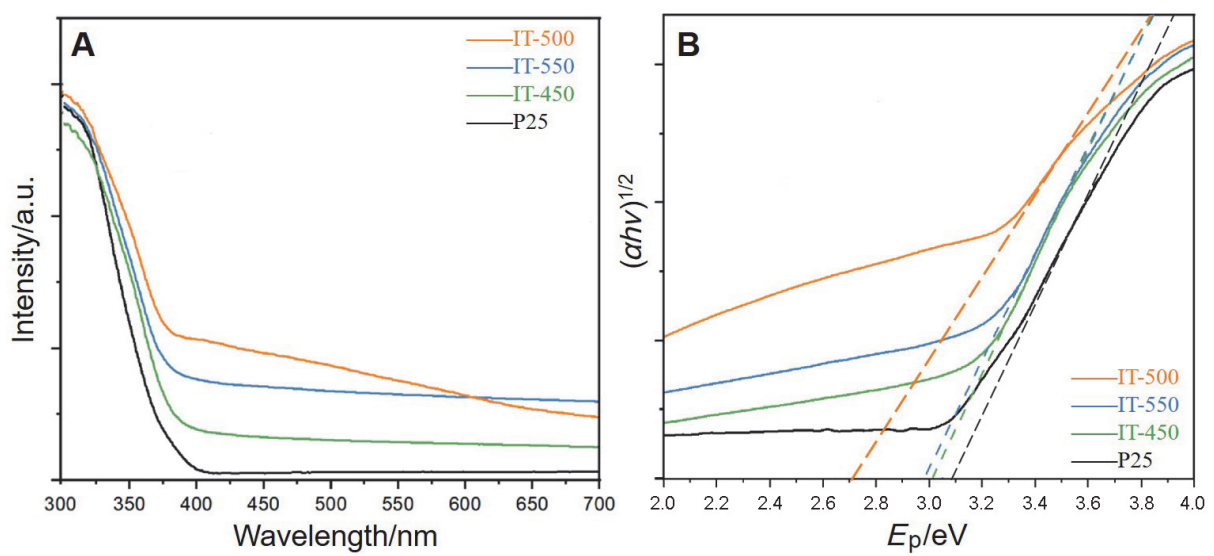

图 5 (A) IT-450, IT-500, IT-550 和 P25 的紫外-可见吸收光谱图; (B) Tauc 图用于确定带隙

Figure 5 (A) UV-vis absorption spectra of IT-450, IT-500, IT-550 and P25; (B) Tauc plot for band gap determination. 
利用光致发光(PL)谱图测量光生载流子的二次结 合发光的光强, 来表征不同样品中光生载流子的复合 率. 如图 6 所示, P25 的 PL 光谱强度最高, 表明纯的 $\mathrm{TiO}_{2}$ 中, 电子和空穴比较容易发生复合, 而经过改性的 样品的 PL 光谱强度均低于纯 $\mathrm{TiO}_{2}$, 证明 $\mathrm{In}_{2} \mathrm{O}_{3} / \mathrm{TiO}_{2}$ 异 质结的引入, 在两相界面处形成了接触电场, 促进了电 子空穴对的分离与转移, 抑制了电荷载流子的复合 ${ }^{[44]}$. 基于上述表征, 表明我们已经制备了预期的 $\mathrm{N}, \mathrm{B}$ 共掺 杂的 $\mathrm{In}_{2} \mathrm{O}_{3} / \mathrm{TiO}_{2}$ 异质结结构.

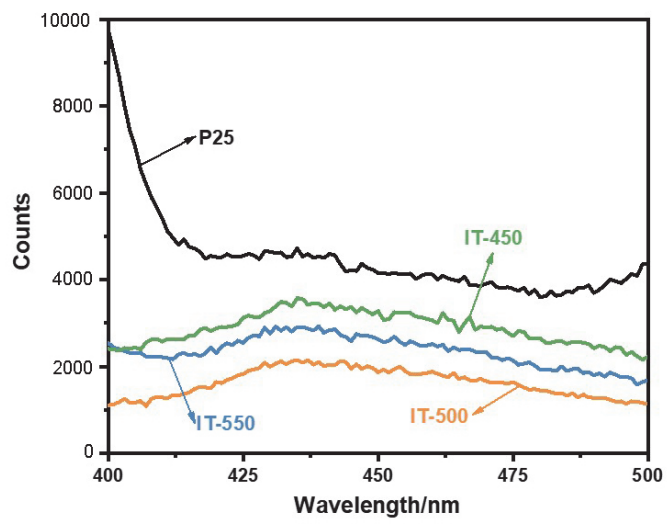

图 6 IT-450, IT-500 和 IT-550 的光致发光光谱图

Figure 6 Photoluminescence spectra of IT-450, IT-500 and IT-550

\section{$2.4 \mathrm{~B}, \mathrm{~N}-\mathrm{In}_{2} \mathrm{O}_{3} / \mathrm{TiO}_{2}$ 的光催化产氢性能}

进一步研究了 IT-450, IT-500, IT-550 与 P25 的光催 化产氢活性, 使用 Pt 纳米粒子作为助催化剂, 三乙醇胺 (TEOA) 作为空穴牺牲剂. 如图 7A 所示, 负载 Pt 纳米颗 粒的 IT-450、IT-500、IT-550 在波长大于 $380 \mathrm{~nm}$ 的可见 光照射下, 光催化产氢速率分别为 3839、5961、5709 $\mu \mathrm{mol} \cdot \mathrm{g}^{-1} \cdot \mathrm{h}^{-1}$, 而同实验条件下的 P25 仅有 $135 \mu \mathrm{mol} \cdot$ $\mathrm{g}^{-1} \cdot \mathrm{h}^{-1}$. 通过对比前文的表征结果, 相比其他样品, IT-500 有着更大的比表面积、更窄的带隙以及更宽的吸 收光谱范围, 并且在 IT-500 中, 光生载流子发生复合的 概率要小于另外两种样品. 选择产氢活性最高的 IT-500 进行循环实验, 如图 7B 所示, 在经过 5 次循环后, 所制 备催化剂的光催化产氢活性未发生明显变化.

将 $\mathrm{B}, \mathrm{N}$ 共掺杂 $\mathrm{In}_{2} \mathrm{O}_{3} / \mathrm{TiO}_{2}$ 聚合物前驱体利用气纺丝 制备得到 $\mathrm{B}, \mathrm{N}$ 掺杂 $\mathrm{In}_{2} \mathrm{O}_{3} / \mathrm{TiO}_{2}$ 纤维棉. 纤维棉的实物图 与 SEM 电镜图如图 8 所示, 纤维呈蓬松纤维棉状, 有一 定的回弹性. 纤维表面较为粗糙, 利于增加其比表面积, 提高光催化效率. 各元素在纤维表面均匀分布, 其 EDS 面扫结果见图 S4.

在 $500{ }^{\circ} \mathrm{C}$ 的退火条件下, 该体系纤维的光催化产 氢速率可达到 $1186 \mu \mathrm{mol} \cdot \mathrm{g}^{-1} \cdot \mathrm{h}^{-1}$, 在经过 5 次循环实验 后仍能达到初始产氢速率的 $97 \%$, 其 5 次循环数据如图 9 所示. 由于催化剂整体为一个团状, 在进行光催化反 应过程中可维持宏观形态, 可以在实验后轻易地从体系 中分离回收, 进行循环重复使用. 较文献中 $\mathrm{TiO}_{2}$ 纳米纤
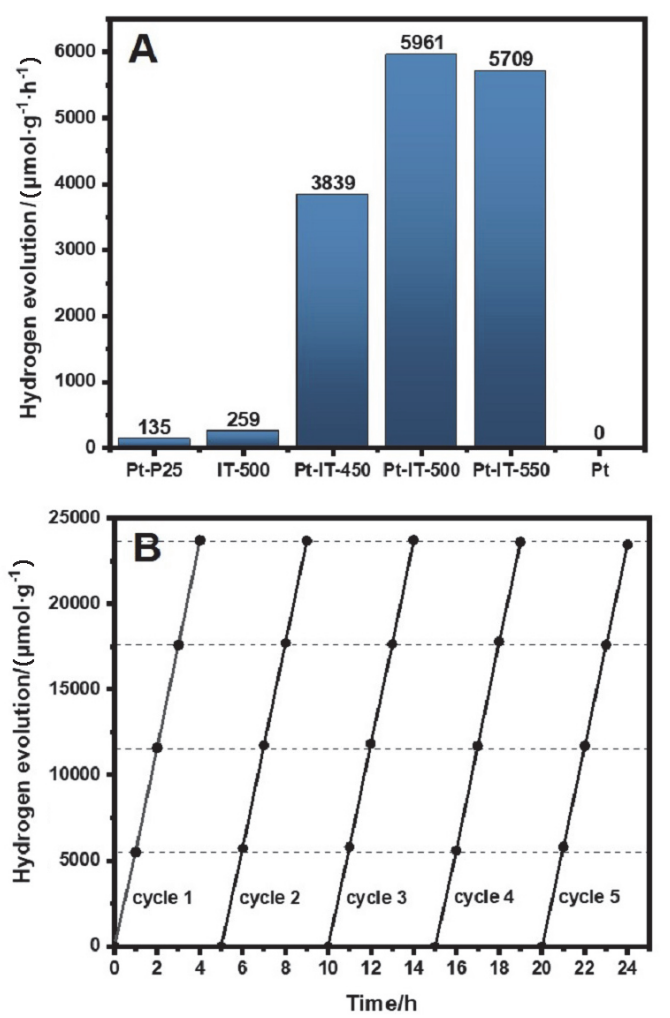

图 7 (A) P25, IT-450, IT-500 和 IT-550 的光催化产氢速率; (B) IT-500 的光催化产氢循环实验

Figure 7 (A) Photocatalytic hydrogen production rates of P25, IT-450, IT-500 and IT-550; (B) photocatalytic hydrogen production cycle experiments of IT-500

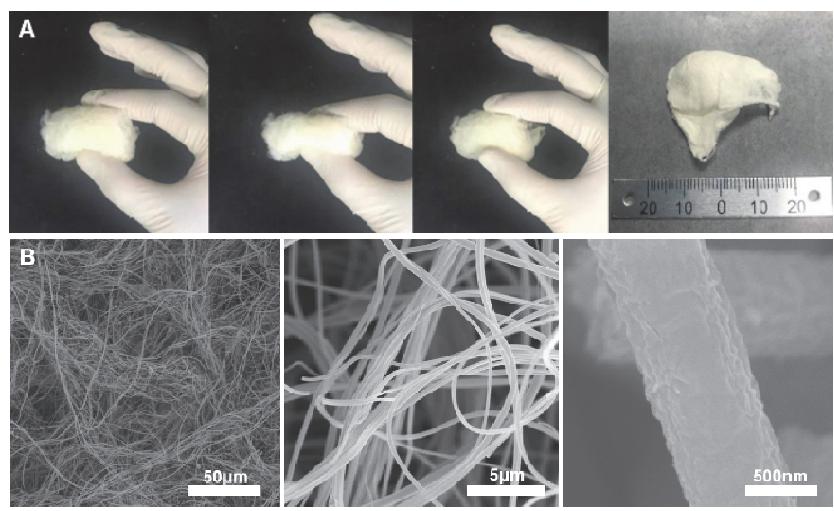

图 $8 \mathrm{~B}, \mathrm{~N}-\mathrm{In}_{2} \mathrm{O}_{3} / \mathrm{TiO}_{2}$ 纤维实物图(A)和 SEM 图(B)

Figure 8 Picture of real products (A) and SEM images (B) of B, $\mathrm{N}-\mathrm{In}_{2} \mathrm{O}_{3} / \mathrm{TiO}_{2}$ fibers

维的产氢速率亦有明显提高(见表 S4).

\section{$2.5 \mathrm{~B}, \mathrm{~N}-\mathrm{In}_{2} \mathrm{O}_{3} / \mathrm{TiO}_{2}$ 的光催化产氢机理}

根据表征结果分析，我们推测了 $\mathrm{N}, \mathrm{B}$ 掺杂的 $\mathrm{In}_{2} \mathrm{O}_{3} / \mathrm{TiO}_{2}$ 的反应机理, 如图 10 所示. 由于非金属元素 $\mathrm{N}$ 和 $\mathrm{B}$ 的掺杂，在价带顶部生成了局部中间态，使得 $\mathrm{TiO}_{2}$ 的带隙变窄，从而将样品对太阳光的吸收范围拓宽 至可见光区域, 这提高了样品对于太阳光的吸收利用 


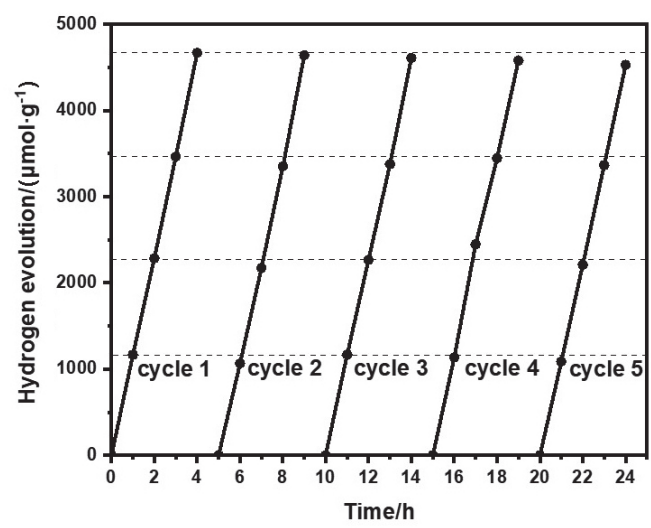

图 $9 \mathrm{~B}, \mathrm{~N}-\mathrm{In}_{2} \mathrm{O}_{3} / \mathrm{TiO}_{2}$ 纳米纤维的光催化产氢循环实验

Figure 9 Photocatalytic hydrogen production cycle experiments of $\mathrm{B}$, $\mathrm{N}-\mathrm{In}_{2} \mathrm{O}_{3} / \mathrm{TiO}_{2}$ nanofibers.

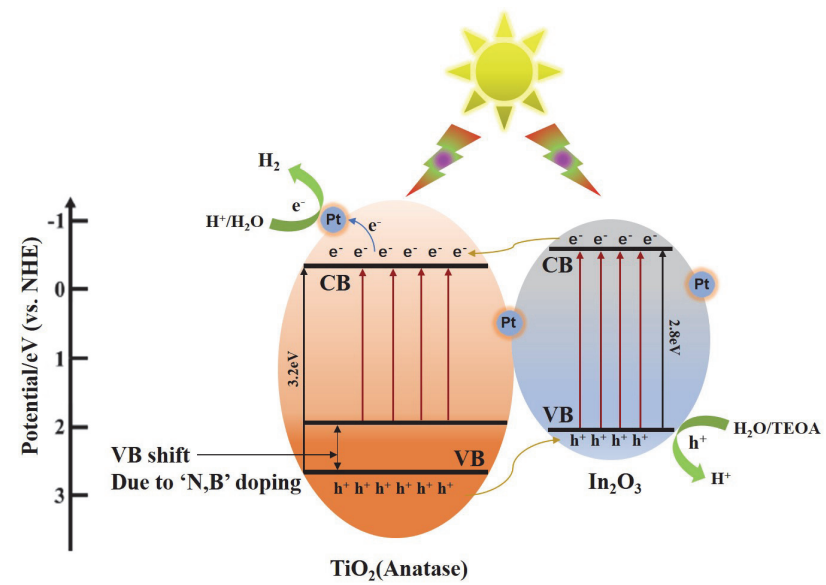

图 $10 \mathrm{~N}, \mathrm{~B}$ 掺杂的 $\mathrm{In}_{2} \mathrm{O}_{3} / \mathrm{TiO}_{2}$ 的光催化机理示意图

Figure 10 Schematic illustration of photocatalysis mechanism of N, B co-doped $\mathrm{In}_{2} \mathrm{O}_{3} / \mathrm{TiO}_{2}$

率; 异质结结构的存在促进了激子的分离, 减少了它们 发生复合的概率. 具体而言, 当样品在可见光的照射下, 改性 $\mathrm{TiO}_{2}$ 和 $\mathrm{In}_{2} \mathrm{O}_{3}$ 吸收光激发后会产生电子-空穴对, 由 于 $\mathrm{TiO}_{2}$ 的导带能级比 $\mathrm{In}_{2} \mathrm{O}_{3}$ 更负, 因此 $\mathrm{In}_{2} \mathrm{O}_{3}$ 所产生的电 子会转移至 $\mathrm{TiO}_{2}$ 的导带上, 同时由于较低的价带电势, 在 $\mathrm{TiO}_{2}$ 中产生的空穴也将迁移至 $\mathrm{In}_{2} \mathrm{O}_{3}$ 的价带, 促进激 子分离为自由电荷, 使得更多电子和空穴分别参与到还 原与氧化反应中. 另外, 由于助催化剂 $\mathrm{Pt}$ 纳米粒子的负 载, 形成界面肖特基势垒, 来自 $\mathrm{TiO}_{2}$ 或 $\mathrm{In}_{2} \mathrm{O}_{3} \mathrm{CB}$ 的电子 可以通过肖特基势垒迁移到金属中, 并且金属可以储存 电子, 形成反应位点, 还原 $\mathrm{H}^{+}$生成 $\mathrm{H}_{2}$; 金属 $\mathrm{Pt}$ 具有较 高的功函数, 转移至金属的光生电子将无法重新回流至 受激半导体, 促进了载流子的分离与转移, 同时贵金属 助催化剂可以降低产生氢气的过电位 ${ }^{[29]}$.

\section{3 结论}

本工作报道了一种通过多种改性手段协同作用来 增强 $\mathrm{TiO}_{2}$ 基光催化剂活性的方法. $\mathrm{PEG}$ 作为致孔剂引入
体系，在煅烧裂解后形成介孔结构，提高了光催化剂的 比表面积. $\mathrm{B}, \mathrm{N}$ 共掺杂可以使 $\mathrm{TiO}_{2}$ 的带隙变窄, 拓宽了 $\mathrm{TiO}_{2}$ 的光谱吸收范围, 使其可见光吸收范围显著拓宽. $\mathrm{In}_{2} \mathrm{O}_{3} / \mathrm{TiO}_{2}$ 作为异质结结构可以有效地分离激子为自由 电荷, 使电子和空穴分别参与还原和氧化反应, 减少它 们复合的概率, 提高了光催化效率. 在光催化产氢实验 中, 利用光沉积法负载了 $\mathrm{Pt}$ 纳米颗粒, 在大于 $380 \mathrm{~nm}$ 的可见光照射下, 粉末状光催化剂 IT-500 的光催化产氢 速率达到了 $5961 \mu \mathrm{mol} \cdot \mathrm{g}^{-1} \cdot \mathrm{h}^{-1}$, 远优于 P25 和大部分使 用单一改性手段制备的 $\mathrm{TiO}_{2}$ 基光催化剂, 在此基础上 利用所合成的聚合物前驱体溶液, 通过气纺丝装置制备 成纤维棉状催化剂, 测试得其光催化产氢速率达到 $1186 \mu \mathrm{mol} \cdot \mathrm{g}^{-1} \cdot \mathrm{h}^{-1}$, 在经过 5 次循环实验后, 仍能达到 初始产氢速率的 97\%. 纤维棉状催化剂在分离回收过程 中可以缩短时间周期, 简化操作步骤, 具有经济性与环 保性，在大规模制备、高效稳定使用方面具有巨大潜力.

\section{4 实验部分}

\section{1 制备 $\mathrm{B}, \mathrm{N}-\mathrm{In}_{2} \mathrm{O}_{3} / \mathrm{TiO}_{2}$}

采用聚合物前驱体法制备了 $\mathrm{B}, \mathrm{N}$ 共掺杂的 $\mathrm{In}_{2} \mathrm{O}_{3} / \mathrm{TiO}_{2}$ 光催化剂. 将 $92.6 \mathrm{~g}$ 铁酸异丙酯、 $39 \mathrm{~g}$ 的 PEG $\left(M_{\mathrm{n}}=600\right)$ 与 $1.23 \mathrm{~g}$ 硝酸铟混合, 在搅拌下升温至 $120{ }^{\circ} \mathrm{C}$, 保温 $2 \mathrm{~h}$. 降温至 $90{ }^{\circ} \mathrm{C}$ 后, 加入 $4.82 \mathrm{~g}$ 嗍酸, 升 温至 $120{ }^{\circ} \mathrm{C}$ 保温 $2 \mathrm{~h}$, 降温至 $90{ }^{\circ} \mathrm{C}$ 后加入 $38.39 \mathrm{~g}$ 乙酰 胺, 升温至 $120{ }^{\circ} \mathrm{C}$ 保温 $2 \mathrm{~h}$. 冷却至 $85{ }^{\circ} \mathrm{C}$, 加入 $9.75 \mathrm{~g}$ 乙酰丙酮, 随后使用恒压漏斗以 $1 \sim 2$ 滴/秒的速度滴入 $5.85 \mathrm{~g}$ 水和 $23.4 \mathrm{~g}$ 正丙醇的混合物进行可控水解聚合, 滴加完成后回流 $1 \mathrm{~h}$, 旋蒸除去溶剂, 得到 $\mathrm{B}, \mathrm{N}$ 共掺杂 的 $\mathrm{In}_{2} \mathrm{O}_{3} / \mathrm{TiO}_{2}$ 聚合物前驱体. 将该前驱体放置于石英管 式炉中, 以 $3{ }^{\circ} \mathrm{C} \cdot \mathrm{min}^{-1}$ 升至预定温度 $\left(450,500,550{ }^{\circ} \mathrm{C}\right)$, 保温 $30 \mathrm{~min}$, 随后将产物放入研钭中研磨成粉体, 得到 $\mathrm{B}, \mathrm{N}$ 共掺杂的 $\mathrm{In}_{2} \mathrm{O}_{3} / \mathrm{TiO}_{2}$ 催化剂.

$\mathrm{B}, \mathrm{N}$ 掺杂的 $\mathrm{In}_{2} \mathrm{O}_{3} / \mathrm{TiO}_{2}$ 纳米纤维的制备: 以聚乙烯 吡咯烷酮 $\left(\mathrm{PVP}, M_{\mathrm{n}}=1300000\right)$ 为纺丝助剂, 以乙醇和乙 酸为溶剂, 将前驱体、PVP、乙醇、乙酸按照质量比 1 : $1: 3: 1$ 均匀混合成纺丝液; 以压缩空气为气源, 采用 气纺丝装置将纺丝液从针头中挤出, 利用高流速气体将 纺丝液拉伸成纳米纤维形态, 进气气压为 $0.10 \mathrm{MPa}$, 进 料速度为 $40 \mathrm{~mL} \cdot \mathrm{h}^{-1}$, 接收距离为 $50 \mathrm{~cm}$, 使用笼状装置 收集纳米纤维. 随后经过湿气固化、在 $500{ }^{\circ} \mathrm{C}$ 下焙烧 30 $\min$, 获得 $\mathrm{B}, \mathrm{N}$ 掺杂的 $\mathrm{In}_{2} \mathrm{O}_{3} / \mathrm{TiO}_{2}$ 纳米纤维.

\section{2 光催化产氢性能测试}

将 $10 \mathrm{mg}$ 光催化剂, $5 \mathrm{~mL}$ 水, $1 \mathrm{~mL}$ 三乙醇胺(TEOA) 加入 $25 \mathrm{~mL}$ 的试管中, 然后加入 $30 \mu \mathrm{L}$ 氯铂酸水溶液(氯 铂酸浓度为 $10 \mathrm{mg} \cdot \mathrm{mL}^{-1}$ ), 通过光沉积法沉积催化剂质 量分数 $3 \%$ 的 Pt 纳米粒子作为助催化剂. 将体系真空处 理几次, 并用氩气鼓泡 $30 \mathrm{~min}$ 以除去溶解的空气. 光催 化产氢反应在 $300 \mathrm{~W}$ 氙灯(截止波长 $380 \mathrm{~nm}$ ) 的可见光照 
射下开始，该灯配有冷却系统以保持温度. 反应 $4 \mathrm{~h}$ 后， 通过气相色谱仪(GC, HP4890D)分析产生氢气的量, 该 色谱仪配备了以氦气作为内标的 FID 和 TCD 检测器.

\section{References}

[1] Fujishima, A.; Honda, K. Nature 1972, 238, 37.

[2] Li, X.; Zhang, T. Y.; Wang, T.; Zhao, Y. X. Acta Chim. Sinica 2019, 77,1075 (in Chinese). (李金鍂, 张太阳, 王甜, 赵一新, 化学学报, 2019, 77, 1075.)

[3] Yu, H.; Ye, L.; Zhang, T.; Zhou, H.; Zhao, T. RSC Adv. 2017, 7, 15265.

[4] Yu, H.; Chen, F.; Ye, L.; Zhou, H.; Zhao, T. J. Mater. Sci. 2019, 54, 10191.

[5] Long, H. J.; Wang, E. J.; Dong, J. Z.; Wang, L. L.; Cao, Y. Q.; Yang, W. S.; Cao, Y. A. Acta Chim. Sinica 2009, 67, 1533 (in Chinese). (龙 绘锦, 王恩君, 董江舟, 王玲玲, 曹永强, 杨文胜, 曹亚安, 化学 学报, 2009, 67, 1533.)

[6] Guo, Y.; Li, Y. R.; Wang, C. M.; Long, R.; Xiong, Y. J. Acta Chim. Sinica 2019, 77, 520 (in Chinese). (郭宇, 李燕瑞, 王成名, 龙再, 熊宇杰, 化学学报, 2019, 77, 520.)

[7] Chen, X. B.; Liu, L.; Yu, P. Y.; Mao, S. S. Science 2011, 331, 746.

[8] Marchal, C.; Cottineau, T.; Mendez-Medrano, M. G.; ColbeauJustin, C.; Caps, V.; Keller, V. Adv. Energy Mater. 2018, 8, 1702142.

[9] Peng, Z. K.; Ding, H. M.; Chen, R. F.; Gao, C.; Wang, C. Acta Chim. Sinica 2019, 77, 681 (in Chinese). (彭正康, 丁慧敏, 陈如凡, 高 超, 汪成, 化学学报, 2019, 77, 681.)

[10] Perillo, P. M.; Rodríguez, D. F. J. Alloys Compd. 2016, 657, 765.

[11] Seo, M.-H.; Yuasa, M.; Kida, T.; Huh, J.-S.; Yamazoe, N.; Shimanoe, K. Sensor. Actuat. B-Chem. 2011, 154, 251.

[12] Asahi, R.; Morikawa, T.; Ohwaki, T.; Aoki, K.; Taga, Y. Science 2001, 293, 269.

[13] Schneider, J.; Matsuoka, M.; Takeuchi, M.; Zhang, J.; Horiuchi, Y.; Anpo, M.; Bahnemann, D. W. Chem. Rev. 2014, 114, 9919.

[14] Yang, H. G.; Liu, G.; Qiao, S. Z.; Sun, C. H.; Jin, Y. G.; Smith, S. C.; Zou, J.; Cheng, H. M.; Lu, G. Q. J. Am. Chem. Soc. 2009, 131, 4078.

[15] Erwin, W. R.; Zarick, H. F.; Talbert, E. M.; Bardhan, R. Energy Environ. Sci. 2016, 9, 1577.

[16] Kumaravel, V.; Mathew, S.; Bartlett, J.; Pillai, S. C. Appl. Catal. B-Environ. 2019, 244, 1021.

[17] Chai, Z. ; Zeng, T. T.; Li, Q.; Lu, L. Q.; Xiao, W. J.; Xu, D. J. Am. Chem. Soc. 2016, 138, 10128.

[18] Huang, H.; Jin, Y.; Chai, Z.; Gu, X.; Liang, Y.; Li, Q.; Liu, H.; Jiang, H.; Xu, D. Appl. Catal. B-Environ. 2019, 257, 117869.

[19] Di Valentin, C.; Pacchioni, G.; Selloni, A.; Livraghi, S.; Giamello, E. J. Phys. Chem. B 2005, 109, 11414.

[20] Geng, H.; Yin, S.; Yang, X.; Shuai, Z.; Liu, B. J. Phys. Condens. Matter 2006, $18,87$.

[21] Liu, G.; Zhao, Y.; Sun, C.; Li, F.; Lu, G. Q.; Cheng, H. M. Angew. Chem. Int. Ed. 2008, 47, 4516.

[22] Finazzi, E.; Di Valentin, C.; Pacchioni, G. J. Phys. Chem. C 2009,
113,3382 .

[23] Sakthivel, S.; Kisch, H. Angew. Chem. Int. Ed. 2003, 42, 4908.

[24] Di Valentin, C.; Pacchioni, G.; Selloni, A. Chem. Mater. 2005, 17, 6656.

[25] Huang, D.-G.; Liao, S.-J.; Liu, J.-M.; Dang, Z.; Petrik, L. J. Photochem. Photobiol., A 2006, 184, 282.

[26] Yu, J. C.; Yu, J. G.; Ho, W. K.; Jiang, Z. T.; Zhang, L. Z. Chem. Mater. 2002, 14, 3808.

[27] Park, H.; Choi, W. J. Phys. Chem. B 2004, 108, 4086.

[28] Bidaye, P. P.; Khushalani, D.; Fernandes, J. B. Catal. Lett. 2009, 134, 169.

[29] Marschall, R. Adv. Funct. Mater. 2014, 24, 2421.

[30] Li, X.; Zhou, X.; Guo, H.; Wang, C.; Liu, J.; Sun, P.; Liu, F.; Lu, G. ACS Appl. Mater. Interfaces 2014, 6, 18661.

[31] Wang, M.; Han, J.; Xiong, H.; Guo, R. Langmuir 2015, 31, 6220.

[32] He, Q.; Sun, H.; Shang, Y.; Tang, Y.; She, P.; Zeng, S.; Xu, K.; Lu, G.; Liang, S.; Yin, S.; Liu, Z. Appl. Surf. Sci. 2018, 441, 458.

[33] Hu, W.; Zhou, W.; Zhang, K.; Zhang, X.; Wang, L.; Jiang, B.; Tian, G.; Zhao, D.; Fu, H. J. Mater. Chem. A 2016, 4, 7495.

[34] Ding, D.; Liu, K.; He, S.; Gao, C.; Yin, Y. Nano Lett. 2014, 14, 6731.

[35] Gao, Y. M.; Shen, H. S.; Dwight, K.; Wold, A. Mater. Res. Bull. 1992, 27, 1023 .

[36] Zhang, X.; Lei, L. J. Hazard. Mater. 2008, 153, 827.

[37] Zhang, L.; Wang, L.; Wei, Y.; Zhang, M.; Jiang, H.; Li, J.; Li, S.; Li, J. Eur. J. Inorg. Chem. 2015, 2015, 5039.

[38] Soares, G. B.; Bravin, B.; Vaz, C. M. P.; Ribeiro, C. Appl. Catal. B-Environ. 2011, 106, 287.

[39] Tan, Y.; Shu, Z.; Zhou, J.; Li, T.; Wang, W.; Zhao, Z. Appl. Catal. B-Environ. 2018, 230, 260.

[40] Wang, X.; Zhang, J.; Wang, L.; Li, S.; Liu, L.; Su, C.; Liu, L. J. Mater. Sci. Technol. 2015, 31, 1175.

[41] Yang, Y.; Liang, Y.; Wang, G.; Liu, L.; Yuan, C.; Yu, T.; Li, Q.; Zeng, F.; Gu, G. ACS Appl. Mater. Interfaces 2015, 7, 24902.

[42] Cavalcante, R. P.; Dantas, R. F.; Bayarri, B.; González, O.; Giménez, J.; Esplugas, S.; Machulek, A. Catal. Today 2015, 252, 27.

[43] Pujilaksono, B.; Klement, U.; Nyborg, L.; Jelvestam, U.; Hill, S.; Burgard, D. Mater. Charact. 2005, 54, 1.

[44] Mu, J.; Chen, B.; Zhang, M.; Guo, Z.; Zhang, P.; Zhang, Z.; Sun, Y.; Shao, C.; Liu, Y. ACS Appl. Mater. Interfaces 2012, 4, 424.

[45] Cong, Y.; Zhang, J.; Chen, F.; Anpo, M. J. Phys. Chem. C 2007, 111, 6976.

[46] Xing, M.-Y.; Li, W.-K.; Wu, Y.-M.; Zhang, J.-L.; Gong, X.-Q. J. Phys. Chem. C 2011, 115, 7858.

[47] Patel, N.; Jaiswal, R.; Warang, T.; Scarduelli, G.; Dashora, A.; Ahuja, B. L.; Kothari, D. C.; Miotello, A. Appl. Catal. B-Environ. 2014, 150-151, 74.

[48] Ling, Q.; Sun, J.; Zhou, Q. Appl. Surf. Sci. 2008, 254, 3236.

[49] Feng, N.; Zheng, A.; Wang, Q.; Ren, P.; Gao, X.; Liu, S.-B.; Shen, Z.; Chen, T.; Deng, F. J. Phys. Chem. C 2011, 115, 2709.

[50] Zhang, K.; Wang, X.; He, T.; Guo, X.; Feng, Y. Powder Technol. 2014, 253, 608 .

(Cheng, B.) 